\title{
The Correlation Between Serum Adipokines and Liver Cell Damage in Non-Alcoholic Fatty Liver Disease
}

\author{
Raika Jamali, ${ }^{1}$ Neda Hatami, ${ }^{2}$ and Farid Kosari $^{3, *}$ \\ ${ }^{1}$ Research Development Center, Digestive Disease Research Institute, Sina Hospital, Tehran University of Medical Sciences, Tehran, IR Iran \\ ${ }^{2}$ Sina Hospital, Tehran University of Medical Sciences, Tehran, IR Iran \\ ${ }^{3}$ Department of Pathology, Sina Hospital, Tehran University of Medical Sciences, Tehran, IR Iran \\ "Corresponding author: Farid Kosari, Department of Pathology, Sina Hospital, Tehran University of Medical Sciences, Tehran, IR Iran. Tel: +98-9123435346, E-mail: \\ faridkosari@yahoo.com
}

Received 2016 February 25; Revised 2016 March 07; Accepted 2016 March 18.

\begin{abstract}
Background: Non-alcoholic fatty liver disease (NAFLD) is a common cause of chronic hepatitis, which can lead to cirrhosis and hepatocellular carcinoma.

Objectives: The aim of the study was to evaluate the correlation between serum adipocytokines and the histologic findings of the liver in patients with non-alcoholic fatty liver disease (NAFLD).

Patients and Methods: This case-control study was performed on those with persistent elevated liver enzymes and with evidence of fatty liver in ultrasonography. After exclusion of patients with other etiologies causing abnormal liver function tests, the resulting patients underwent liver biopsies. NAFLD was diagnosed based on liver histology according to the Brunt scoring system.

Results: Waist circumferences and levels of blood glucose (after fasting), insulin, triglycerides, alanine aminotransferases (ALT), and aspartate aminotransferases (AST) were higher in patients with NAFLD than in those in the control group. ALT, AST, and gamma glutamine transferase (GGT) levels were lower in patients with liver steatosis of a grade of less than $33 \%$ than those with higher degrees of steatosis. Serum low-density lipoprotein (LDL), cholesterol, and hepcidin levels were significantly higher in those with lobular inflammation of grade 0 - 1 than in those with inflammation of grade 2 - 3 (Brunt score). Meanwhile, AST was significantly lower in those with lobular inflammation of grade 1 than in those with grade 2-3. Hepcidin and resistin levels were significantly higher in patients with moderate to severe fibrosis than in those with mild fibrosis.

Conclusions: It seems that surrogate liver function tests and adipocytokine levels were correlated with the histologic findings of the liver.
\end{abstract}

Keywords: Non-Alcoholic Fatty Liver Disease, Liver Biopsy, Visfatin, Resistin, Hepcidin

\section{Background}

Non-alcoholic fatty liver disease (NAFLD) is a common cause of chronic hepatitis, which can lead to cirrhosis and hepatocellular carcinoma $(1,2)$. This disease is considered to be a representation in the liver of insulin resistance or metabolic syndrome. The prevalence of the disease is increasing, which is mainly due to its close relationship with obesity and diabetes mellitus (3). NAFLD is increasingly becoming known as the main cause of liver-related mortality, and insulin resistance is a key factor in the development of a fatty liver (4).

Liver ultrasonography is usually used as a screening tool in diagnosing NFLD. The precision of the method depends on the sonographer; however, the method itself cannot detect slight changes in liver fat. Therefore, this method cannot be used in follow-up checks of NAFLD patients. Moreover, this method cannot differentiate between fibrosis and fatty changes (5).
The exact pathogenesis of the disease and the progression mechanisms are not clearly known. However, it is worth noting that insulin resistance and the role of adipocytokines secreted from the visceral adipose tissue have been taken into serious consideration in the course of disease pathogenesis (3). Studies have been conducted on adiponectin and leptin (6-8), but few studies have examined other adipocytokines such as visfatin, resistin, and hepcidin. Considering the role of the mentioned biomarkers in the pathogenesis of NAFLD as discussed in previous studies, it seems reasonable to consider that their relationship with the extent of liver damage may be in line with the common serum levels in the patients.

Clinical, imaging and laboratory findings are restricted in their ability to predict the severity of the disease. Liver biopsy is the gold standard of diagnostic methods and the best indicator of the extent of liver damage; nevertheless, possible complications due to its invasive nature have placed limitations on its application. 


\section{Objectives}

The present study aims at examining the relationship between the new specialized biomarkers of NAFLD and liver tissue pathologic findings.

\section{Patients and Methods}

This case-control study was conducted on patients more than 18 years of age who were referred to the gastroenterology clinic of a general hospital. Patients with constant increases in liver enzymes along with evidence of a fatty liver in sonography were enrolled in the study. Those with positive viral markers ( $\mathrm{HBc} \mathrm{Ab}, \mathrm{HBs} \mathrm{Ag}, \mathrm{HCV}$ $\mathrm{Ab}$ ), indications of metabolic disorder (decreased serum ceruloplasmin, transferrin saturation $>45 \%$ ), evidence of autoimmune hepatitis (including serum gamma globulin $>1.2 \mathrm{~g}$ (dL and positive FANA), daily alcohol consumption $>20 \mathrm{~g}$, hepatotoxic medication in the last three months, chronic disabling diseases (e.g., heart failure, chronic obstructive pulmonary disease, cirrhosis, chronic renal failure), or any form of cancer were excluded from the study. Liver biopsy was performed in the remaining patients for confirmation of NAFLD. The possible complications of this diagnostic method, as well as the steps involved in conducting the study, were explained to the patients. After obtaining informed consent, liver biopsies were performed.

In order to study steatosis/inflammation and fibrosis in the samples, hematoxylin-eosin staining and trichrome and reticulin stains were used. Liver tissue samples with at least five portal spaces were considered appropriate and were studied according to the Brunt scoring system (9). One single pathologist studied the liver biopsies to prevent inter-observer variability bias in interpreting the findings. Certain people were selected from among those referring to the GI Clinic with reasons other than liver problems and companies of the patients. The case and control groups' ages and sexes were matched by an epidemiologist. The control group had normal liver enzymes and ultrasonography with no evidence of liver diseases in their physical examinations and laboratory tests. Taking into account 5\% type I error (alpha) and 95\% power with reference to the previous studies, 18 persons were assigned to each group.

Waist circumference $(\mathrm{cm})$ and BMI (weight $/ \mathrm{m}^{2}$ ) were measured for all patients. Levels of fasting blood sugar (FBS), insulin, alanine aminotransferases (ALT), aspartate aminotransferases (AST), alkaline phosphatases (ALP), gamma-glutamyl transferases (GGT), triglycerides (TG), cholesterol, and high and low-density lipoproteins (HDL and LDL), as well as serum levels of visfatin, resistin, and hepcidin, were measured in the blood samples using the enzyme-linked immunosorbent assay (ELISA).
The mean ( \pm standard deviation) was determined for all quantitative data. A t-test was used to compare the mean values of the studied variables between cases and controls. Binary logistic regression was applied for evaluating the correlation between the factors under study and the findings of the liver biopsies. The data were statistically analyzed using SPSS, ver. 17 . A P value $<0.05$ was considered significant.

This study was approved by the ethics committee of the Tehran University of Medical Sciences (registration No.: 9111160013).

\section{Results}

The mean age of the participants in the study was 34.50 ( \pm 8.85 ) years (range: 19 - 50 years). The results of studying the demographic and laboratory variables are presented in Table 1.

There were 13 (72\%) patients with less than 33\% and five $(28 \%)$ with more than $33 \%$ steatosis in the liver biopsy. Grade 0 - 1 lobular inflammation was observed in 9 (50\%) patients, and the other half had lobular inflammation of grade $2-3$. Mild fibrosis was found in 11 (61\%) patients, and 7 (39\%) had moderate or severe fibrosis.

Clinical and laboratory findings (mean \pm SD) among cases with hepatocyte steatosis $<33 \%$ and $>33 \%$, lobular inflammation of grade $0-1$ and grade $2-3$, as well as mild and moderate/severe fibrosis are demonstrated in Table 2. The correlation between steatosis, inflammation, and liver fibrosis in NAFLD patients with clinical and laboratory indications can also be seen in Table 3 .

\section{Discussion}

According to the results of this study, the waist circumferences and levels of insulin, TG, ALT, and AST of the patients in the NAFLD group were higher than the numbers of those in the control group. Serum levels of LDL and hepcidin in lobular inflammation of grade 0/1 was significantly higher than the levels among those in the inflammation grade 2/3 group. Meanwhile, the serum levels of AST in the lobular inflammation grade $0 / 1$ group were considerably lower than those in the grade $2 / 3$ group. Furthermore, the serum levels of hepcidin and resistin in the moderate/severe liver fibrosis group were remarkably higher than the numbers in those with cases of mild fibrosis. However, no relationship was found between the laboratory findings including adipocytokines and the variables studied in the liver pathology according to the multivariate correlation study. Nevertheless, one of the advantages of the present study was the presence of liver biopsy data 
Table 1. Clinical Specifications and Laboratory Findings in the Studied Groups ${ }^{a}$

\begin{tabular}{|c|c|c|c|}
\hline $\begin{array}{l}\text { Demographic } \\
\text { and Laboratory } \\
\text { Variables }\end{array}$ & $\begin{array}{l}\text { Control Group (18 } \\
\text { Persons) }\end{array}$ & $\begin{array}{l}\text { NAFL Group (18 } \\
\text { Persons) }\end{array}$ & P Value \\
\hline Age & $30.44 \pm 10.11$ & $34.50 \pm 8.85$ & 0.20 \\
\hline Sex & & & - \\
\hline Male & $13(72)$ & $13(72)$ & \\
\hline Female & $5(28)$ & $5(28)$ & \\
\hline $\begin{array}{l}\text { Waist } \\
\text { circumference, } \\
\text { cm }\end{array}$ & $99.5 \pm 2.28$ & $102.72 \pm 2.94$ & 0.001 \\
\hline BMI, $\mathrm{kg} / \mathrm{m}^{2}$ & $29.28 \pm 3.89$ & $31.58 \pm 3.94$ & 0.087 \\
\hline FBS, $\mathrm{mg} / \mathrm{dL}$ & $90.50 \pm 5.46$ & $104.33 \pm 12.28$ & 0.001 \\
\hline Insulin, mU/L & $7.64 \pm 2.95$ & $12.91 \pm 5.09$ & 0.001 \\
\hline TG, mg/dL & $81.07 \pm 23.39$ & $129.63 \pm 57.13$ & 0.002 \\
\hline $\begin{array}{l}\text { Cholesterol, } \\
\text { mg/dL }\end{array}$ & $156.56 \pm 33.19$ & $165.52 \pm 26.38$ & 0.37 \\
\hline HDL, mg/dL & $47.48 \pm 20.12$ & $45.66 \pm 7.45$ & 0.59 \\
\hline LDL, $\mathbf{m g} / \mathbf{d L}$ & $92.86 \pm 23.27$ & $96.71 \pm 23.04$ & 0.62 \\
\hline ALT, U/L & $25.11 \pm 15.68$ & $57.61 \pm 35.67$ & 0.001 \\
\hline AST, $\mathbf{U} / \mathbf{L}$ & $24.47 \pm 5.59$ & $39.48 \pm 17.40$ & 0.001 \\
\hline ALP, $\mathbf{U} / \mathbf{L}$ & $166.10 \pm 34.59$ & $147.69 \pm 22.92$ & 0.06 \\
\hline $\mathbf{G G T}, \mathbf{U} / \mathbf{L}$ & $35.33 \pm 22.16$ & $34.76 \pm 25.65$ & 0.94 \\
\hline Visfatin, ng/mL & $12.45 \pm 13.42$ & $20.67 \pm 15.96$ & 0.10 \\
\hline Hepcidin, ng/mL & $0.77 \pm 0.42$ & $0.83 \pm 0.90$ & 0.79 \\
\hline Resistin, mg/mL & $2.66 \pm 1.17$ & $2.06 \pm 1.10$ & 0.12 \\
\hline \multicolumn{4}{|c|}{$\begin{array}{l}\text { Abbreviations: ALP, alkaline phosphatases; ALT, alanine aminotransferases; AST, } \\
\text { aspartate aminotransferases; BMI, body mass index; FBS, fasting blood sugar; } \\
\text { GGT, gamma-glutamyl transferases; HDL, high density lipoproteins; LDL, low } \\
\text { density lipoproteins; TG, triglycerides. } \\
\text { a Values are expressed as mean } \pm \text { SD or No. (\%). }\end{array}$} \\
\hline
\end{tabular}

through which the severity of NAFLD and the relationship with the level of adipocytokines and the other mentioned parameters was determined.

The present study showed no meaningful relationship between the serum levels of visfatin and liver fibrosis severity. This was contrary to the findings of the previous study conducted on patients with morbid obesity and NAFLD; the increased visfatin level was observed in the liver for all cases, and was significantly higher in patients with fibrosis (10).

The same study showed no relationship between steatosis, inflammatory activity of the liver, and visfatin (10). In this study, the rate of visfatin in steatosis $<33 \%$ was higher than steatosis $>33 \%$, but the difference was not statistically significant. However, in a study (11) designed for measuring visfatin levels in the adipose tissue of NAFLD patients, visfatin levels were high in the non-NAFLD group and reduced in the mild steatosis, moderate steatosis, and non-alcoholic steatohepatitis groups, respectively. Reduction in mild steatosis demonstrates a reverse relationship between visfatin levels and the incidence of mild steatosis in non-alcoholic fatty liver.

The findings of this study confirmed the previous results of another study stating that visfatin levels decrease in non-alcoholic steatohepatitis (12). The reduction has also been observed in more severe clinical stages of liver diseases and cirrhosis (13). In another study (14), it was found that visfatin plasma levels can predict the presence of portal inflammation in NAFLD patients. Our findings are compatible with the previous study (15) in which there was no meaningful relationship between visfatin levels in both the case and control groups; moreover, visfatin levels were not correlated with the pathologic stages of fatty liver.

The resistin difference was not statistically meaningful between the case and control groups. In addition, resistin levels and the liver pathologic findings were not related. However, in a previous study (16), blood resistin levels and BMIs of the NAFLD group were evidently higher than the corresponding numbers of the control. Also, the percentage of liver steatosis and BMI were associated with blood resistin levels. In addition, in a study evaluating the relationship between serum resistin and NAFL (4), resistin levels were higher in the NAFLD group compared to the levels in the control group. Nevertheless, no relationship was found between resistin and highly-sensitive CRP, BMI, and levels of insulin, glucose, transaminases, and lipids.

It is noteworthy that in this study, there was a direct relationship between resistin and the pathologic inflammatory scores. Similar results were obtained from a study conducted on obese patients undergoing bariatric surgery (17). In this study, adiponectin instead of resistin predicted the development of non-alcoholic steatohepatitis. In previous studies $(18,19)$, higher resistin levels were in line with more severe stages of fatty liver fibrosis. In our study, resistin levels were higher in the moderate/severe fibrosis group as compared to the rates of those with mild fibrosis.

In this study, serum hepcidin levels were correlated with inflammation and liver fibrosis. These findings are similar to the previous ones (20) in which hepcidin serum levels were obviously higher in NAFLD patients. Also, it was found that hepcidin serum levels were directly associated with total cholesterol and triglyceride levels, but not with iron indices and the pathologic characteristics of NAFLD.

\subsection{Summary}

In this research, several adipocytokines and serum indicators common in NAFLD evaluation (including metabolic and liver function tests) were simultaneously 
Table 2. Comparison (Mean \pm SD) of Clinical and Laboratory Findings Among Cases With Less or More Than 33\% Steatosis, Lobular Inflammation of Grade One or More, and Mild and More Severe Fibrosis ${ }^{\mathrm{a}}$,,$c$

\begin{tabular}{|c|c|c|c|c|c|c|c|c|c|}
\hline \multirow[t]{2}{*}{ Demographic and Laboratory Variables } & \multicolumn{2}{|c|}{ Steatosis Severity } & \multirow[t]{2}{*}{$\mathbf{P}$} & \multicolumn{2}{|c|}{ Lobular Inflammation Severity } & \multirow[t]{2}{*}{$\mathbf{P}$} & \multicolumn{2}{|c|}{ Fibrosis Severity } & \multirow[t]{2}{*}{$\mathbf{P}$} \\
\hline & $\leq \mathbf{3 3} \%$ & $>33 \%$ & & Grade 2-3 & Grade 0 - 1 & & Moderate or Severe & Mild & \\
\hline Waist circumference, cm & $101 \pm 3.67$ & $103.38 \pm 2.46$ & 0.12 & $102.11 \pm 3.40$ & $103.33 \pm 2.44$ & 0.39 & $103.14 \pm 3.53$ & $102.45 \pm 2.65$ & 0.64 \\
\hline BMI, $\mathrm{kg} / \mathrm{m}^{2}$ & $29.17 \pm 2.96$ & $32.50 \pm 3.97$ & 0.11 & $31.29 \pm 3.64$ & $31.87 \pm 4.42$ & 0.76 & $31.24 \pm 4.72$ & $31.80 \pm 3.59$ & 0.77 \\
\hline FBS, $\mathbf{m g} / \mathbf{d L}$ & $109.60 \pm 18.17$ & $102.31 \pm 9.35$ & 0.27 & $102.44 \pm 13.6$ & $106.22 \pm 11.28$ & 0.53 & $108.29 \pm 16.19$ & $101.82 \pm 9$ & 0.28 \\
\hline Insulin, $\mathrm{mU} / \mathbf{L}$ & $13.02 \pm 5.02$ & $12.87 \pm 5.32$ & 0.95 & $13.89 \pm 5.12$ & $11.93 \pm 5.17$ & 0.43 & $10.85 \pm 4.65$ & $14.22 \pm 5.12$ & 0.17 \\
\hline TG, mg/dL & $131.10 \pm 65.64$ & $129.06 \pm 56.45$ & 0.94 & $144.60 \pm 68.76$ & $114.66 \pm 41.29$ & 0.27 & $102.37 \pm 38.17$ & $146.98 \pm 61.84$ & 0.10 \\
\hline HDL, mg/dL & $41.14 \pm 5.12$ & $47.40 \pm 7.61$ & 0.11 & $45.27 \pm 8.39$ & $46.05 \pm 6.86$ & 0.83 & $46.17 \pm 7.52$ & $45.34 \pm 7.75$ & 0.82 \\
\hline LDL, mg/dL & $98.30 \pm 17$ & $96.05 \pm 25.80$ & 0.86 & $85.53 \pm 21.58$ & $109.30 \pm 18.42$ & 0.02 & $107.25 \pm 17.99$ & $90.97 \pm 24.18$ & 0.17 \\
\hline ALT, $\mathbf{U} / \mathbf{L}$ & $96 \pm 36.55$ & $42.85 \pm 22.53$ & 0.002 & $73.22 \pm 40.71$ & $42.01 \pm 22.34$ & 0.06 & $42.57 \pm 27.93$ & $67.19 \pm 37.90$ & 0.15 \\
\hline AST, $\mathbf{U} / \mathbf{L}$ & $60 \pm 15.74$ & $31.60 \pm 10.19$ & 0.001 & $47.64 \pm 19.95$ & $31.33 \pm 9.79$ & 0.04 & $35.71 \pm 12.13$ & $41.89 \pm 20.26$ & 0.48 \\
\hline ALP, U/L & $150 \pm 29.24$ & $146.80 \pm 21.36$ & 0.80 & $147.03 \pm 26.27$ & $148.35 \pm 20.62$ & 0.90 & $153.42 \pm 18.30$ & $144.04 \pm 25.58$ & 0.41 \\
\hline GGT, U/L & $55.18 \pm 37.33$ & $26.91 \pm 15.06$ & 0.03 & $39.85 \pm 32.7$ & $29.67 \pm 16.45$ & 0.41 & $31.51 \pm 19.06$ & $36.83 \pm 29.80$ & 0.68 \\
\hline Visfatin, ng/mL & $27.10 \pm 13.24$ & $18.02 \pm 16.69$ & 0.30 & $24.07 \pm 14.36$ & $17.26 \pm 17.58$ & 0.38 & $16.57 \pm 14.916$ & $23.28 \pm 16.72$ & 0.40 \\
\hline Hepcidin, ng/mL & $0.42 \pm 0.20$ & $0.99 \pm 1.02$ & 0.23 & $0.34 \pm 0.16$ & $1.33 \pm 1.07$ & 0.01 & $1.46 \pm 1.20$ & $0.43 \pm 0.26$ & 0.01 \\
\hline Resistin, mg/mL & $1.80 \pm 1.18$ & $2.16 \pm 1.10$ & 0.54 & $1.94 \pm 0.91$ & $2.18 \pm 1.31$ & 0.65 & $3.04 \pm 0.95$ & $1.44 \pm 0.66$ & 0.01 \\
\hline
\end{tabular}

Table 3. Correlation Between the Severity of Steatosis, Lobular Inflammation, and Fibrosis Among the Clinical and Laboratory Findings in NAFLD Patients

\begin{tabular}{|c|c|c|c|c|c|c|c|c|c|}
\hline \multirow[t]{2}{*}{ Demographic and Laboratory Variables } & \multicolumn{2}{|c|}{ Steatosis Severity } & \multirow[t]{2}{*}{$\mathbf{P}$} & \multicolumn{2}{|c|}{ Lobular Inflammation Severity } & \multirow[t]{2}{*}{$\mathbf{P}$} & \multicolumn{2}{|c|}{ Fibrosis Severity } & \multirow[t]{2}{*}{$\mathbf{P}$} \\
\hline & Correlation Coefficient & $\mathrm{CI}$ (low, high) & & Correlation coefficient & $\mathrm{CI}$ (low, high) & & Correlation Coefficient & $\mathrm{CI}($ low, high $)$ & \\
\hline Waist circumference, $\mathrm{cm}$ & 0.22 & $-0.56,1.01$ & 0.34 & -0.10 & $-0.93,0.72$ & 0.63 & 0.01 & $-0.60,0.62$ & 0.93 \\
\hline BMI, $\mathrm{kg} / \mathrm{m}^{2}$ & -0.16 & $-0.62,0.29$ & 0.26 & 0.02 & $-0.45,0.50$ & 0.84 & -0.13 & $-0.49,0.22$ & 0.23 \\
\hline FBS, $\mathrm{mg} / \mathrm{dL}$ & 0.01 & $-0.13,0.15$ & 0.79 & 0.01 & $-0.13,0.15$ & 0.81 & -0.02 & $-0.13,0.08$ & 0.49 \\
\hline Insulin, $\mathbf{m U} / \mathbf{L}$ & 0.04 & $-0.23,0.32$ & 0.58 & 0.06 & $-0.23,0.35$ & 0.45 & -0.03 & $-0.25,0.18$ & 0.53 \\
\hline $\mathrm{TG}, \mathrm{mg} / \mathrm{dL}$ & 0.01 & $-0.03,0.03$ & 0.88 & 0.01 & $-0.03,0.04$ & 0.54 & -0.01 & $-0.03,0.02$ & 0.33 \\
\hline HDL, mg/dL & -.0 .02 & $-0.23,0.17$ & 0.61 & 0.01 & $-0.19,0.23$ & 0.73 & -0.06 & $-0.22,0.09$ & 0.20 \\
\hline LDL, mg/dL & 0.01 & $-0.05,0.07$ & 0.67 & -0.01 & $-0.07,0.06$ & 0.82 & -0.01 & $-0.06,0.03$ & 0.35 \\
\hline ALT, $\mathbf{U} / \mathbf{L}$ & -0.01 & $-0.06,0.05$ & 0.61 & -0.01 & $-0.07,0.05$ & 0.64 & -0.01 & $-0.04,0.04$ & 0.99 \\
\hline AST, $\mathbf{U} / \mathbf{L}$ & 0.03 & $-0.06,0.13$ & 0.28 & 0.01 & $-0.08,0.12$ & 0.54 & 0.01 & $-0.07,0.08$ & 0.85 \\
\hline ALP, U/L & -0.01 & $-0.13,0.11$ & 0.76 & -0.01 & $-0.14,0.11$ & 0.66 & 0.01 & $-0.07,0.11$ & 0.50 \\
\hline GGT, U/L & 0.01 & $-0.12,0.12$ & 0.90 & 0.01 & $-0.12,0.13$ & 0.92 & -0.02 & $-0.11,0.07$ & 0.45 \\
\hline Visfatin, ng/mL & -0.01 & $-0.08,0.04$ & 0.37 & -0.01 & $-0.07,0.06$ & 0.80 & -0.01 & $-0.05,0.04$ & 0.65 \\
\hline Hepcidin, ng/mL & -0.23 & $-1.65,1.17$ & 0.54 & -0.39 & $-1.88,1.08$ & 0.36 & 0.31 & $-0.78,1.41$ & 0.34 \\
\hline Resistin, mg/mL & 0.05 & $-1.46,1.56$ & 0.90 & 0.43 & $-1.14,2.01$ & 0.36 & 0.03 & $-1.14,1.20$ & 0.91 \\
\hline
\end{tabular}

Abbreviations: ALP, alkaline phosphatases; ALT, alanine aminotransferases; AST, aspartate aminotransferases; BMI, body mass index; FBS, fasting blood sugar; GGT, gamma-glutamyl transferases; HDL, high density lipoproteins; LDL, low density lipoproteins; TG, triglycerides.

studied to determine if there was any significant association between the biomarkers and the liver pathologic findings. Although there was a meaningful relationship between the serum levels of the biomarkers for the case and control groups in the univariate analysis, the serum levels were not associated with liver pathologic findings in the multivariate analysis.

Since liver biopsies are not welcomed by all patients due to their invasive nature and costs, finding new mark- ers for determining the possible relationship of these variables with the severity of fatty liver pathology can be helpful in effectively diagnosing the stage of the disease. This study can therefore be a start for future studies leading to more effective management and treatment of NAFLD.

It should be noted, however, that the amount of adipokines might fluctuate during the course of NAFLD. Therefore, the results of this case-control study need to be investigated in future prospective studies. Meanwhile, the 
level of adipokines in the visceral adipose tissue and liver seem to have a more important role in the pathogenesis of NAFLD than the serum levels. Measuring the effect of adipokine levels in visceral adipose tissue on liver cell damage could be considered another future direction for research.

\section{Acknowledgments}

The authors would like to thank Fatemeh Esfahani (Epidemiologist) at Tehran University of Medical Sciences for analyzing the data, and Bita Pourmand from the urology research center for translating the article into English.

\section{Footnotes}

Authors' Contribution: Study concept and design: Raika Jamali; acquisition of data: Neda Hatami; analysis and interpretation of data: Raika Jamali, Neda Hatami, and Farid Kosari; drafting of the manuscript: Neda Hatami and Farid Kosari; critical revision of the manuscript for important intellectual content: Farid Kosari and Raika Jamali; statistical analysis: Raika Jamali; administrative, technical, and material support: Raika Jamali and Farid Kosari; study supervision: Raika Jamali and Farid Kosari.

Funding/Support: Funding for this research was supported by Tehran University of Medical Sciences.

\section{References}

1. Duan XY, Qiao L, Fan JG. Clinical features of nonalcoholic fatty liver disease-associated hepatocellular carcinoma. Hepatobiliary Pancreat Dis Int. 2012;11(1):18-27. [PubMed: 22251466].

2. ButtAS, Abbas Z, Jafri W. Hepatocellular carcinoma in pakistan: where do we stand?. Hepat Mon. 2012;12(10 HCC):ee6023. doi: 10.5812/hepatmon.6023. [PubMed: 23166534].

3. Gatselis NK, Ntaios G, Makaritsis K, Dalekos GN. Adiponectin: a key playmaker adipocytokine in non-alcoholic fatty liver disease. Clin Exp Med. 2014;14(2):121-31. doi: 10.1007/s10238-012-0227-0. [PubMed: 23292294].

4. Pagano C, Soardo G, Pilon C, Milocco C, Basan L, Milan G, et al. Increased serum resistin in nonalcoholic fatty liver disease is related to liver disease severity and not to insulin resistance. J Clin Endocrinol Metab. 2006;91(3):1081-6. doi:10.1210/jc.2005-1056. [PubMed: 16394091].

5. Jamali R. Non-Alcoholic Fatty Liver Disease: Diagnosis and Evaluation of Disease Severity. Thrita J Med Sci. 2013;2(2):43-51. doi: 10.5812/thrita.11795.
6. Dongiovanni P, Fracanzani AL, Fargion S, Valenti L. Iron in fatty liver and in the metabolic syndrome: a promising therapeutic target. $J$ Hepatol. 2011;55(4):920-32. doi: 10.1016/j.jhep.2011.05.008. [PubMed: 21718726].

7. Schaffler A, Scholmerich J, Buchler C. Mechanisms of disease: adipocytokines and visceral adipose tissue-emerging role in nonalcoholic fatty liver disease. Nat Clin Pract Gastroenterol Hepatol. 2005;2(6):27380. doi: 10.1038/ncpgasthep0186. [PubMed: 16265231].

8. Polyzos SA, Toulis KA, Goulis DG, Zavos C, Kountouras J. Serum total adiponectin in nonalcoholic fatty liver disease: a systematic review and meta-analysis. Metabolism. 2011;60(3):313-26. doi: 10.1016/j.metabol.2010.09.003. [PubMed: 21040935].

9. Brunt EM, Janney CG, Di Bisceglie AM, Neuschwander-Tetri BA, Bacon BR. Nonalcoholic steatohepatitis: a proposal for grading and staging the histological lesions. Am J Gastroenterol. 1999;94(9):2467-74. doi: 10.1111/j.1572-0241.1999.01377.x. [PubMed:10484010].

10. Kukla M, Ciupinska-Kajor M, Kajor M, Wylezol M, Zwirska-Korczala K, Hartleb M, et al. Liver visfatin expression in morbidly obese patients with nonalcoholic fatty liver disease undergoing bariatric surgery. Pol J Pathol. 2010;61(3):147-53. [PubMed: 21225497].

11. Gaddipati R, Sasikala M, Padaki N, Mukherjee RM, Sekaran A, JayarajMansard M, et al. Visceral adipose tissue visfatin in nonalcoholic fatty liver disease. Ann Hepatol. 2010;9(3):266-70. [PubMed: 20720266].

12. Jarrar MH, Baranova A, Collantes R, Ranard B, Stepanova M, Bennett $\mathrm{C}$, et al. Adipokines and cytokines in non-alcoholic fatty liver disease. Aliment Pharmacol Ther. 2008;27(5):412-21. doi: 10.1111/j.13652036.2007.03586.x. [PubMed: 18081738].

13. de Boer JF, Bahr MJ, Boker KH, Manns MP, Tietge UJ. Plasma levels of PBEF/Nampt/visfatin are decreased in patients with liver cirrhosis. Am J Physiol Gastrointest Liver Physiol. 2009;296(2):G196-201. doi: 10.1152/ajpgi.00029.2008. [PubMed:19074645].

14. Aller R, de Luis DA, Izaola O, Sagrado MG, Conde R, Velasco MC, et al. Influence of visfatin on histopathological changes of non-alcoholic fatty liver disease. Dig Dis Sci. 2009;54(8):1772-7. doi: 10.1007/s10620008-0539-9. [PubMed: 19005759].

15. Genc H, Dogru T, Kara M, Tapan S, Ercin CN, Acikel C, et al. Association of plasma visfatin with hepatic and systemic inflammation in nonalcoholic fatty liver disease. Ann Hepatol. 2013;12(4):548-55. [PubMed: 23813132].

16. Murad A, Hassan H, Husein H, Ayad A. Serum resistin levels in nonalcoholic fatty liver disease and their relationship to severity of liver disease. IEndocrinol Metabol Diabetes South Africa. 2010;15(1):53-5.

17. Baranova A, Gowder SJ, Schlauch K, Elariny H, Collantes R, Afendy $A$, et al. Gene expression of leptin, resistin, and adiponectin in the white adipose tissue of obese patients with non-alcoholic fatty liver disease and insulin resistance. Obes Surg. 2006;16(9):1118-25. doi: 10.1381/096089206778392149. [PubMed: 16989692].

18. Elsayed EY, Mohamed AS, Elal HA, Hamed E. Diagnostic Role Of Resistin In Nonalchoholic Fatty Liver Disease. Group. 2010;51:9.6.

19. Shen C, Zhao CY, Wang W, Wang YD, Sun H, Cao W. The relationship between hepatic resistin overexpression and inflammation in patients with nonalcoholic steatohepatitis. BMC Gasteroenterol. 2014;14:39.

20. Senates E, Yilmaz Y, Colak Y, Ozturk O, Altunoz ME, Kurt R, et al. Serum levels of hepcidin in patients with biopsy-proven nonalcoholic fatty liver disease. Metab Syndr Relat Disord. 2011;9(4):287-90. doi: 10.1089/met.2010.0121. [PubMed: 21417913]. 